\title{
Convex Hull Asymptotic Shape Evolution
}

\author{
Maxim Arnold, Yuliy Baryshnikov and Steven M. LaValle \\ University of Illinois, Urbana, IL 61801, USA; (MA: Institute for Information Transmission \\ Problems, Moscow, Russia). Email: \{mda,ymb,lavalle $\} @$ uiuc.edu
}

\begin{abstract}
Summary. The asymptotic properties of Rapidly exploring Random Tree (RRT) growth in large spaces is studied both in simulation and analysis. The main phenomenon is that the convex hull of the RRT reliably evolves into an equilateral triangle when grown in a symmetric planar region (a disk). To characterize this and related phenomena from flocking and swarming, a family of dynamical systems based on incremental evolution in the space of shapes is introduced. Basins of attraction over the shape space explain why the number of hull vertices tends to reduce and the shape stabilizes to a regular polygon.
\end{abstract}

\section{Introduction}

Rapidly exploring Random Trees (RRTs) [9] have become increasing popular as a way to explore high-dimensional spaces for problems in robotics, motion planning, virtual prototyping, computational biology, and other fields. The experimental successes of RRTs have stimulated interest in their theoretical properties. In [10], it was established that the vertex distribution converges in probability to the sampling distribution. It was also noted that there is a "Voronoi bias" in the tree growth because the probability that a vertex is selected is proportional to the volume of its Voronoi region. This causes aggressive exploration in the beginning, and gradual refinement until the region is uniformly covered. The RRT was generalized so that uniform random samples are replaced by any dense sequence to obtain deterministic convergence guarantees that drive dispersion (radius of the largest empty ball) to zero [11]. The lengths of RRT paths and their lack of optimality was studied in two recent works. Karaman and Frazzoli prove that RRTs are not asymptotically optimal and propose RRT*, which is a variant that converges to optimal path lengths [5]. Nechushtan, Raveh and Halperin developed an automaton-based approach to analyzing cases that lead to poor path quality in RRTs [12]. More broadly, there has also been interest in characterizing convergence rates for other sampling-based planning algorithms that use random sampling [3, 4, 8].

In this paper, we address one of the long-standing questions regarding RRT behavior. Figure 1 (a) shows how an RRT appears in a square region after 390 iterations. 


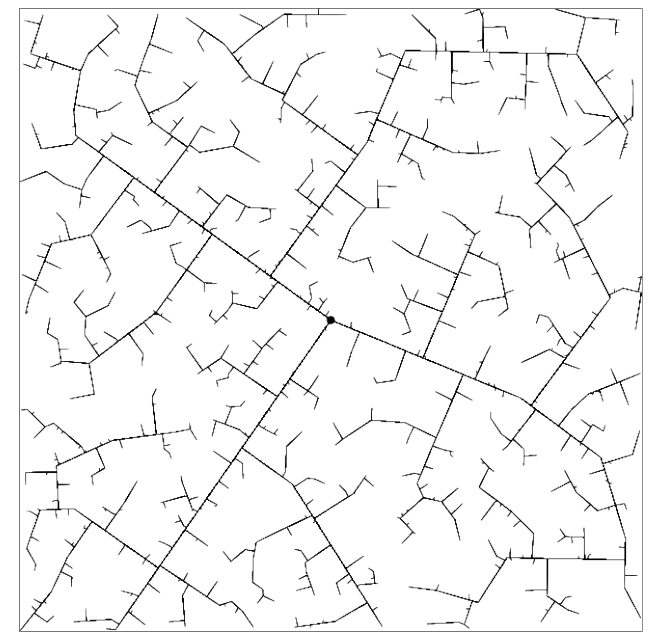

(a)

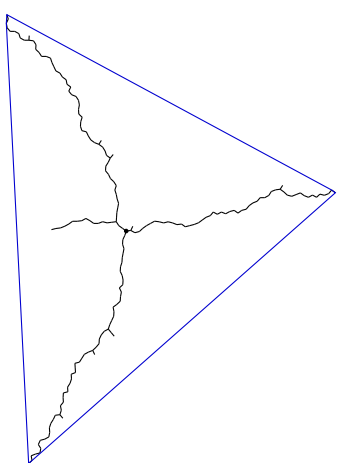

(b)

Fig. 1. (a) An RRT after 390 iterations. (b) An RRT grown from the center of a "large" disc, shown with its convex hull.

Fig. 2. The Voronoi diagram of RRT vertices contains interior Voronoi regions, which are bounded by other Voronoi regions, and exterior Voronoi regions, which extend to the boundary of the space.

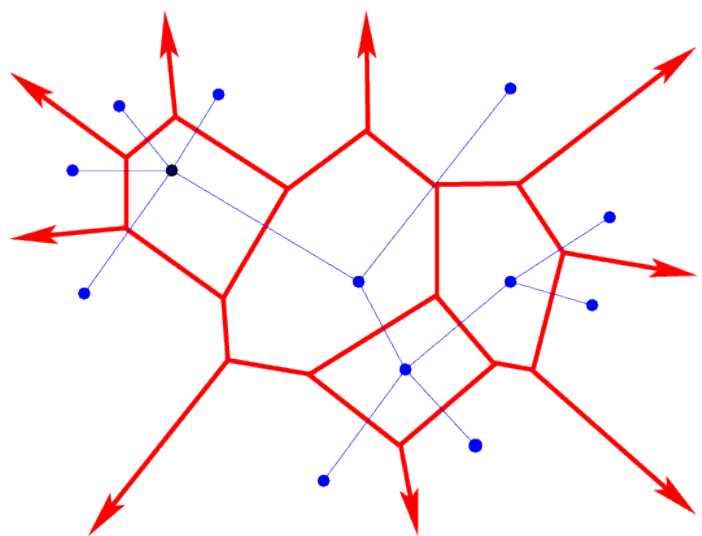


What happens when the size of the search space is increased? In the limiting case in which an RRT sequence is generated in a large disc in the plane, simulations reliably produce three straight tree branches, roughly 120 degrees apart. See Figure 1.b). In terms of Voronoi bias, the exterior Voronoi regions completely dominate because the probability that random samples fall into interior Voronoi regions shrinks to zero; see Figure 1. Thus, the RRT spends virtually all of its time expanding, rather than refining in areas it has already explored.

It is remarkable that the convex hull of the RRT throughout the process is approximately an equilateral triangle with random initial orientation. This has been consistently observed through numerous simulations, and it known for over a decade with no rigorous analysis. How can this behavior be precisely characterized? Why does it occur? This motivates our introduction of convex hull asymptotic shape evolution, CHASE, which is a family of dynamical systems that includes the RRT phenomenon just described.

In addition to understanding RRTs, we believe that the study of CHASE dynamics encompasses a broader class of problems. Another, perhaps comparable in importance, motivation for our research comes from the the general desire to understand the self-organization in large, loosely interacting collectives of agents, whether natural, or artificial. While the literature on flocking, swarming and general dynamics of large populations is immense, it is worth noticing that it is predominantly concentrated on the locally interacting agents. Recently, a new trend emerged, dealing with agents interacting over arbitrary distances: as an example, [14] deals with the scale-invariant rendezvous protocols in swarms, while naturalists provide us with the evidence that scale-invariant (topological, in their parlance) interactions in animal world [1].

\section{Dynamics of Shapes}

\subsection{Rapidly exploring trees}

The Rapidly exploring Random Tree (RRT) is an incremental algorithm that fills a bounded, convex region $X \subset \mathbb{R}^{d}$ in the following way. Let $T(V, E)$ denote a rooted tree embedded in $X$ so that $V \subset X$ and every $e \in E$ is a line segment (with $e \subseteq X$ ). An infinite sequence $\left(T_{0} \subset T_{1} \subset T_{2} \subset \ldots\right)$ of trees is constructed as follows. For $T_{0}\left(V_{0}, E_{0}\right)$, let $E_{0}=\emptyset$ and $V_{0}=\left\{x_{\text {root }}\right\}$ for any chosen $x_{\text {root }} \in X$, designated as the root. Each $T_{i}$ is then constructed from $T_{i-1}$. Let $\varepsilon>0$ be a fixed step size. Select a point $x_{\text {rand }}$ uniformly at random in $X$ and let $x_{\text {near }}$ denote the nearest point in $T_{i-1}$ (in the union of all vertices and edges). If $x_{\text {near }} \in V_{i-1}$ and $\left\|x_{\text {near }}-x_{\text {rand }}\right\| \leq \varepsilon$, then $T_{i}$ is formed by $V_{i}:=V_{i-1} \cup\left\{x_{\text {rand }}\right\}$ and $E_{i}:=E_{i-1} \cup\left\{e_{\text {new }}\right\}$ in which $e_{\text {new }}$ is the segment that connects $x_{\text {near }}$ and $x_{\text {rand }}$. If $\left\|x_{\text {near }}-x_{\text {rand }}\right\|>\varepsilon$, then an edge of length $\varepsilon$ is formed instead, in the direction of $x_{\text {rand }}$, with $x_{\text {new }}$ as the leaf vertex. If $x_{\text {near }} \notin V_{i-1}$, then it must appear in the interior of some edge $e \in E$. In this case, $e$ is split so that both of its endpoints connect to $x_{\text {near }}$. Recall Figure 11a), which shows a sample RRT for $X=[0,1]^{2}$. The RRT described here uses the entire swath 
for selection, rather than vertices alone, as described in [9]. For a discussion of how these two variants are related, see [11]; the asymptotic phenomena are independent of this distinction.

\subsection{Isotropic process}

Consider the situation when the starting point $x_{\text {root }}$ is at the origin, and the region to be explored (and the sampling density) is rotationally invariant. In the limit of small $\varepsilon$, the RRT growth is governed by the direction towards the (far away) $x_{\text {rand }}$, which can be assumed to be uniformly distributed on the unit circle or directions. In this case, the dynamics of the convex hulls of the successive RRTs can be described as follows.

Let $\mathbf{P}_{n}$ denote the convex hull of the points $x_{1}, \ldots, x_{n} \in \mathbb{R}^{2}$. The random polygon $\mathbf{P}_{n+1}$ depends on $x_{1}, \ldots, x_{n}$ only through $\mathbf{P}_{n}$ via the following iterative process:

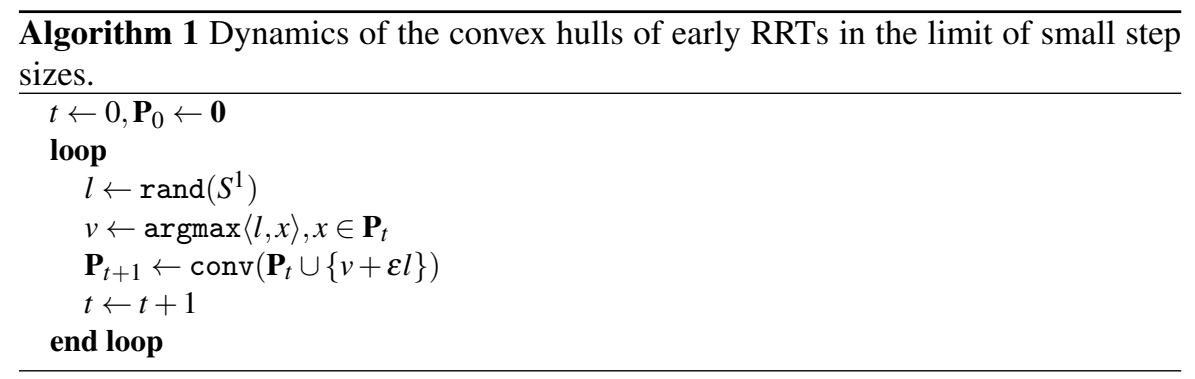

This algorithm defines an increasing family of (random) convex polygons. One might view it as a (growing) Markov process on the space of convex polygons in $\mathbb{R}^{2}$ starting with the origin $\mathbf{P}_{0}$.

It is easy to see that as the Markov chain evolves, the number of vertices in $\mathbf{P}_{n}$ can change. Some vertices can disappear, being swept over by the convex hull of the newly added point. It is easy to see that this new point does not eliminate the vertex $v_{l}$ where the functional $\langle\cdot, l\rangle$ attains its maximum if and only if the vector $-l$ does not belong to the cone $T_{v_{l}^{n}} \mathbf{P}_{n}$, the tangent cone to $\mathbf{P}_{n}$ at $v_{l}$. This, in turn, can happen only if the angle at $v_{l}$ is acute (less than straight).

\section{Enigma of the symmetry breaking}

While the distribution of this growth process is manifestly rotationally invariant, the simulations show that after long enough time the polygons $\mathbf{P}_{n}$ are not getting more and more round. Au contraire, it was observed that the polygons $\mathbf{P}_{n}$ become close (in Hausdorff metrid ${ }^{1}$ ) to some large equilateral triangles. (It should be noted that the

1 Recall that the Hausdorff metric between two subsets of a metric space is defined as $d_{H}(A, B)=\max _{a \in A} \min _{b \in B} d(a, b)+\max _{b \in B} \min _{a \in A} d(a, b)$. 
polygons are actually triangles only for a fraction of the time; typically some highly degenerate - with an interior angle close to $\pi$ - vertices are present.)

While we still do not understand completely the mechanisms of the symmetry breaking and of the formation of the asymptotical shapes, there are several asymptotic results and heuristic models that shed enough light on the process to at least make plausible explanations of the observed dynamics. This note is dealing with these results.

\subsection{Roots of difficulties}

The biggest problem emerging when one attempts to analyze the Markov process $\left\{\mathbf{P}_{n}\right\}_{n=1,2, \ldots}$ stems from the fact that there is no natural parameterization of the space of the polygons: an attempt to account for all convex planar polygons at once leads immediately to an infinite dimensional (and notoriously complicated) space of convex support functions with $C^{0}$ norm-induced topology. Indeed, starting with any polygon, the Markov process will have a transition, with positive probability, changing the collection of vertices forming the convex polygon $\mathbf{P}_{n}$.

Fig. 3. Typical behavior of the number of vertices of $\mathbf{P}_{t}$, starting with a regular 50-sided polygon.

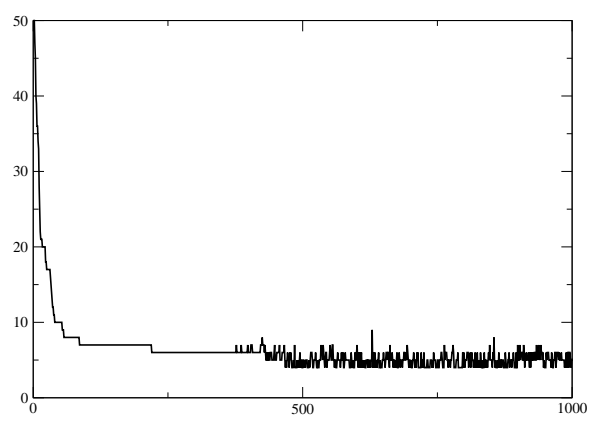

While the experimental evidence suggests that the polygons $\mathbf{P}_{n}$ become close to equilateral triangles in Hausdorff metric, there is little hope to understand the process of the vertices of $\mathbf{P}_{n}$, as the very number of the vertices is changing constantly, see Fig. 2.3

\subsection{Outline of the results}

For this reason, we adopt a circumvential route here. In lieu of addressing the complicated dynamics defined by the algorithm ?? throughout, we concentrate here on 
the time intervals where the number of the vertices of $\mathbf{P}_{t}$ remains constant. During each such stretch, the evolution of the convex polygons $\mathbf{P}$ can be described as a relatively straightforward (time-homogeneous) Markov process on the space of the $k$-sided polygons.

If the number of vertices of polygons $\mathbf{P}_{t}$ were to stabilize, then the size of these polygons would be growing linearly in time (more precisely, the circumference would 2 This suggests to consider a Markov process on the space of shapes of $k$ sided polygons (we discuss the state space of this new process, that is the space of shapes of $k$-sided polygons in section 2.5.

This new Markov process on the space of shapes cannot be time-homogeneous anymore, but would be well approximated by the steps of size $1 / t$. Markov processes on manifolds with diminishing step sizes are quite familiar in the theory of stochastic perturbations, where we represent the Markov process as a shift along a vector field (obtained by the averaging) perturbed by small random noise.

We will describe this averaged dynamical system (which we will refer to as CHASE, for Convex Hull Averaged Stochastic Evolution) in section ??. Interestingly, it can be interpreted (up to a time reparameterization) as the gradient field for the circumference of the polygonal chain: CHASE dynamics tries to make the polygonal chain longer the fastest way!

It is well known that for small steps, the stochastically perturbed processes are tracing closely their deterministic, averaged counterparts for long times. More to the point, the classical results on the stochastic approximation imply that perturbed dynamical system remains trapped with probability one near an exponentially stable equilibrium of the averaged dynamics, if the quadratic deviations of the stochastic perturbation scale like $1 / t$ (or any other scale such that the quadratic variation is summable as $t \rightarrow \infty)$, see [7, 13].

Thus the first natural step to try to explain the lack of polygonal shapes other than equilateral triangles in the long runs of $\mathbf{P}_{t}$ would be to analyze the averaged dynamics of our Markov processes on the polygonal chains, locating their equilibria and studying their stability.

As it turned out, for $k$-sided polygonal chains, the only nondegenerate fixpoints of the averaged dynamics are the regular polygon ${ }^{3}$. Moreover, we prove that these equilibria are unstable for $k \geq 3$.

While the Markov chain $\mathbf{P}_{t}$ is well-approximated by CHASE for $k$-sided polygonal chains with obtuse internal angles, it is not the case in general (because $\mathbf{P}_{t}$ has non-zero probability of changing the number of sides). We present some results that heuristically lend support to the natural conjecture that the regular triangles are stable shapes under any generalized averaged dynamics.

We conclude the paper with a short description of limit of CHASE dynamics for the obtuse $k$-sided polygons, as $k \rightarrow \infty$.

\footnotetext{
${ }^{2}$ We conjecture that this is true in general, but do not have a proof yet.

3 The averaged CHASE dynamics can be extended to non-convex polygons; the regular polygons therefore can be non-convex ones as well.
} 


\subsection{Spaces of shapes}

We begin with the formal description of the space of shapes.

\section{construction}

A configuration of $n$ points is a collection of distinguishable (labelled) points in $\mathbb{R}^{2}$, not all coincident. Traditionally, the shape of a configuration is understood as its class under the equivalence relation on the configurations defined by the Euclidean motions and the change of scale. More specifically, two configurations $\left\{x_{1}, \ldots, x_{n}\right\}$ and $\left\{x_{1}^{\prime}, \ldots, x_{n}^{\prime}\right\}$ are said to have the same shape if for some rotation $U \in S O(2)$, the vector $v \in \mathbb{R}^{2}$ and $\lambda>0, x_{j}^{\prime}=\lambda U x_{j}+v$. Factorings by the scale and the displacement admit sections: one can assume that the center of gravity of the configuration is at the origin, while it moment of inertia is 1 (or that $\sum_{k}\left|x_{k}\right|^{2}=1$ ) - this is where the condition that not all points coincide is important. For a natural interpretation of the space shape in terms of symplectic reduction, see [6].

\section{Complex projective spaces}

Perhaps most explicit way to think about the spaces of shapes of planar $k$-configurations is the following: viewing $\mathbb{R}^{2}$ as $\mathbb{C}$, we can interpret the $k$-configuration with the center of mass at the origin as a point in $\mathbb{C}^{k-1}-\{0\}$. Factoring by rotations and rescaling is equivalent to factoring by $\mathbb{C}_{*}$, the multiplicative group of complex numbers, whence the space of shapes is isomorphic (as a Riemannian manifold) to $\mathbb{C} \mathbb{P}^{k-2}$, the $(k-2)$-dimensional complex projective space.

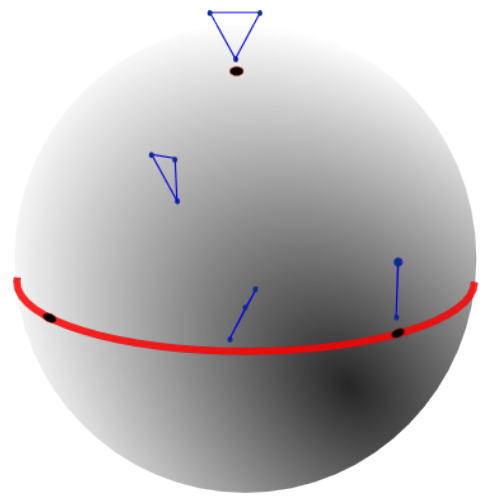

Fig. 4. Sphere of shapes of triangles. The equator consists of degenerate triangles (with three points collinear). The poles correspond to equilateral triangles. 
The simplest nontrivial case $k=3$ is very instructive: $\mathbb{C P}^{1}$ is isometric to the Riemannian sphere $S^{2}$. The poles of the sphere can be identified with the equilateral triangles (North or South depending on the orientation defined by the cyclic order of $x_{1}, x_{2}, x_{3}$ ); the equator corresponds to the collinear triples, and the parallels to the level sets of the (algebraic) area, considered as the function of the configurations rescaled to the moment of inertia 1 (see Figure 4).

\section{Tame configurations and the $k$-gon dynamics}

The space of planar $k$-configurations contains an open subset consisting of tame ones, which we define here as the configurations for which any two consecutive sides form the positively oriented frame (in particular, all points of the configuration are distinct), and the angle between these vectors is acute:

$$
\left(x_{k}-x_{k-1}\right) \times\left(x_{k+1}-x_{k}\right)>0,\left(x_{k}-x_{k-1}\right) \cdot\left(x_{k+1}-x_{k}\right)>0 .
$$

The space of tame configurations is invariant with respect to rotations and dilatations, and its image in the shape space $\operatorname{conf}_{k}=\mathbb{C P}^{k-2}$ is an open contractible set (say, for $k=3$ it is the upper hemisphere of $S^{2}$ ). We will denote the space of tame $k$ configurations as conf ${ }_{k}^{+}$, and the corresponding subset of the shape space as $\operatorname{conf}_{k}^{+}$.

The following observation is obvious: If $\mathbf{P}_{t} \in \operatorname{conf}_{k}^{+}$then for the step size $\varepsilon$ small enough $\mathbf{P}_{t+1} \in \operatorname{conf}_{k}^{+}$. Equivalently, the Markov dynamics on large enough ${ }^{4}$ configuration preserves tameness, for a while.

As one of our goals is to disprove that the Markov process $\mathbf{P}_{t}$ can ever converge to a $k>4$-sided polygon, we can just define a new Markov process which would couple with $\mathbf{P}_{t}$ on tame configurations.

We define the tamed Markov process $\mathbf{P}_{t}^{(k)}$ is defined by the algorithm

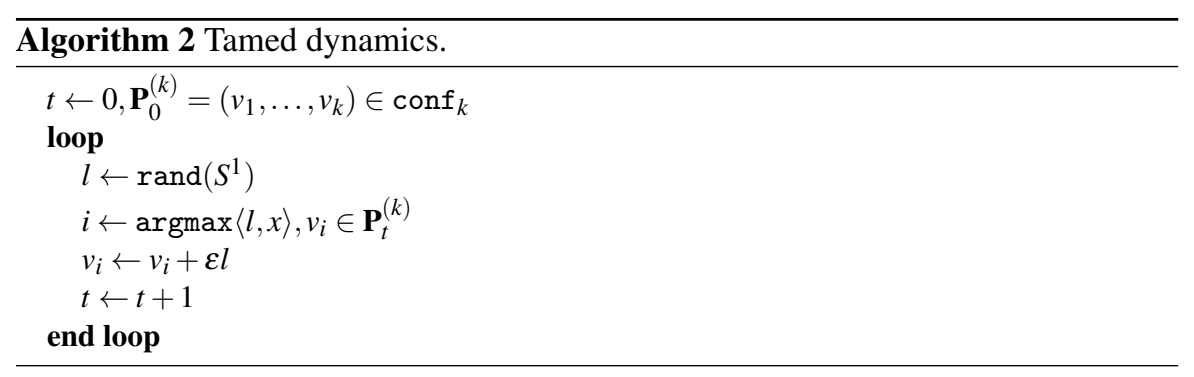

\section{Tamed Dynamics}

The tamed Markov process possesses several features that simplify its treatment considerably, compared to the original Markov process. First and foremost, the tamed

\footnotetext{
${ }^{4}$ And tame enough!
} 
dynamics stays, by definition, in the space of $k$-sided polygonal chains, isomorphic to $\mathbb{R}^{2 k}$.

Further, the fact that the number of the "growth points" is bounded implies, immediately, that the size of the polygonal chain grows as $\Theta(t)$.

Denote by $P(\mathbf{P})$ the perimeter of (not necessarily convex) polygon $\mathbf{P}$.

Lemma 1 The expected perimeter of $\mathbf{P}^{(k)}$ grows linearly: for some $c, C>0$

$$
\mathbb{P}\left(P\left(\mathbf{P}^{(k)}{ }_{t}\right)<c n\right) \leq \exp (-C t) .
$$

(Remark that a linear upper bound on the perimeter of $\mathbf{P}^{(k)}{ }_{t}$ and of $\mathbf{P}_{t}$ is quite immediate.)

\subsection{Continuous dynamics}

The stochastic process $\mathbf{P}^{(k)}$ can be viewed as a deterministic flow on the space $V_{\mathbf{P}}^{k}$ of $k$-sided polygons subject to small noise. Indeed, by the Lemma 1 the size of the polygons $\mathbf{P}^{(k)}$ grows linearly, whence the relative size of the steps decreases.

Given a polygonal $k$-chain $\mathbf{P}$, consider the expected increment

$$
\Delta \mathbf{P}:=\mathbb{E}\left(\mathbf{P}^{(k)}{ }_{t+1} \mid \mathbf{P}^{(k)}{ }_{t}=\mathbf{P}\right)-\mathbf{P}
$$

(we use here the linear structure on $V_{\mathbf{P}}^{k}$ ). The following is immediate:

Lemma 2 The function $\Delta$ commutes with the rotations and is homogeneous of degree 0 .

The linear growth of the sizes of polygons $\mathbf{P}^{(k)}$ together with the homogeneity of $\Delta$ indicate that asymptotically, the expected increments are small compared to the size of $\mathbf{P}^{(k)}$. In such a situation, an approximation of the discrete stochastic dynamics by a continuous deterministic one is a natural step. This motivates the following

Definition 1 The vector field

$$
\dot{\mathbf{P}}=\Delta \mathbf{P}
$$

on $V_{\mathbf{P}}^{k}$ is called the CHASE dynamics.

In coordinates, the CHASE dynamics is given as follows.

Corollary 1 Assume that $\mathbf{P}$ consists of the vertices $\left(x_{1}, \ldots, x_{k}\right), x_{i} \in \mathbb{R}^{2}$ in cyclic or der, and $\left(x_{i_{1}}, x_{i_{2}}, \ldots, x_{i_{c}}\right)$ are the vertices of $\mathbf{P}$ on its convex hull. Then

$$
\dot{x_{i_{l}}}=\frac{x_{i_{l}}-x_{i_{i+1}}}{\left|x_{i_{l}}-x_{i_{i+1}}\right|}+\frac{x_{i_{l}}-x_{i_{i-1}}}{\left|x_{i_{l}}-x_{i_{i-1}}\right|},
$$

and $\dot{x}_{i}=0$ if $x_{i}$ is not on the convex hull of $\mathbf{P}$. 


\subsection{Theorem: discrete converges to continuous}

The intuitive proximity of the $\mathbf{P}^{(k)}$ dynamics and the trajectories of CHASE flow can be made rigorous, using the standard results. Specifically, we have

Theorem 1 Let $\mathbf{P} \in V_{\mathbf{P}}^{k}$, and $\mathbf{P}^{(k)}{ }_{t}, 0 \leq t \leq C \lambda$ be the (random) trajectory of the tamed Markov dynamics with the initial configuration $\lambda \mathbf{P}$. Similarly, let $\mathbf{P}(t), 0 \leq$ $t \leq \lambda^{C}$ be the trajectory of the CHASE dynamics starting with the same initial point $\lambda \mathbf{P}$. Then for any $C>0$, the trajectory $\mathbf{P}^{(k)} / \lambda$ converges to $\mathbf{P}(t) / \lambda$ in probability in $C_{0}$ norm, as $\lambda \rightarrow \infty$.

The proof requires some relatively extensive setup and will not be presented here. However, it is conceptually very transparent: the deviations of the stochastic dynamics from the continuous one has linearly growing quadratic variations, and, by the martingale large deviation results (using, for example, Azuma's inequality), one can prove the proximity of the trajectories for the times of order $\lambda$ with probability exponentially close to 1 .

In other words, the shape of the tamed process follows CHASE dynamical system.

\section{stochastic approximation}

The approach is, of course, close to many classical treatment of the stochastically perturbed dynamical systems, see e.g.[7]. It should be noted, that if one rescales the polygons $\mathbf{P}^{(k)}$ by $t$ (to keep their linear sizes approximately constant), then the step sizes become $c / t$, which is the typical scale of the algorithms of stochastic approximation (compare [13]).

\section{Properties of CHASE Flow}

We outline several properties of the CHASE dynamics, before analyzing their equilibria.

\subsection{Symmetries}

One of the attractive features of the CHASE dynamics is its high symmetries. Thus, the Lemma2 2 implies

Proposition 1 The CHASE dynamics defines a field of directions (i.e. a vector field defined up to point-dependent rescaling) on the shape space $V_{\mathbf{P}}^{k}$.

(We will be retaining the name for the field of direction on $V_{\mathbf{P}}^{k}$ obtained by the projection of CHASE.)

This means that we can track the shapes of the polygonal chains $V_{\mathbf{P}}^{k}$. In particular, if there existed an exponentially stable critical point of the reduction of the 
CHASE dynamics to the shape space, the tamed process would have a positive probability of converging to the corresponding shape.

However, as we will show below, there are no stable equilibria in the space $V_{\mathbf{P}}^{k}$ for $k \geq 4$.

\section{CHASE as the gradient for perimeter}

Another interesting property of CHASE is the fact that it is a gradient flow, with respect to the natural Euclidean metric on $V_{\mathbf{P}}^{k}$.

Proposition 2 The CHASE dynamics is the gradient of the function $\psi(\mathbf{P})$ taking a polygonal chain to the perimeter of its convex hull.

The proof is immediate: the variation of the length of the side $\left[x_{i_{l}}, x_{i_{l+1}}\right]$ when $x_{i_{l}}$ changes infinitesimally is the scalar product with

$$
\frac{x_{i_{l}}-x_{i_{i+1}}}{\left|x_{i_{l}}-x_{i_{i+1}}\right|}
$$

whence the claim follows.

We remark here that if one chooses the other bisector

$$
\dot{x_{i_{l}}}=\frac{x_{i_{l}}-x_{i_{i+1}}}{\left|x_{i_{l}}-x_{i_{i+1}}\right|}-\frac{x_{i_{l}}-x_{i_{i-1}}}{\left|x_{i_{l}}-x_{i_{i-1}}\right|},
$$

as the direction of the velocity, the resulting dynamics would preserve the perimeter and is useful in building customized Birkhoff's billiard tables, see [2].

\subsection{Equilibria}

According to Proposition 1 CHASE defines a vector field on $V_{\mathbf{P}}^{k}$, and one would like to understand which shapes are preserved by this dynamics. Quite obviously, the regular $k$-polygons are preserved. Three more classes of shapes are preserved as well, in fact, shown below: trapezoids, rhombi and drops obtained from a regular $2 k$-polygon by extending a pair of next-to-opposite sides.

As it turned out, these classes exhaust all possible shapes invariant under the CHASE dynamics.

Theorem 2 The equilibrium points of CHASE in conf consist of the polygonal chains whole convex hulls (having $l \leq k$ vertices) are regular $l$-sided polygons.

Proof. Via straightforward trigonometry (using the notation shown on the Figure 6 we derive the rate of change of the angle $\phi_{j}$ as given by

$$
\dot{\varphi}_{j}=\frac{\sin \varphi_{j+1}-\sin \varphi_{j}}{\ell_{j}}+\frac{\sin \varphi_{j-1}-\sin \varphi_{j}}{\ell_{j-1}},
$$



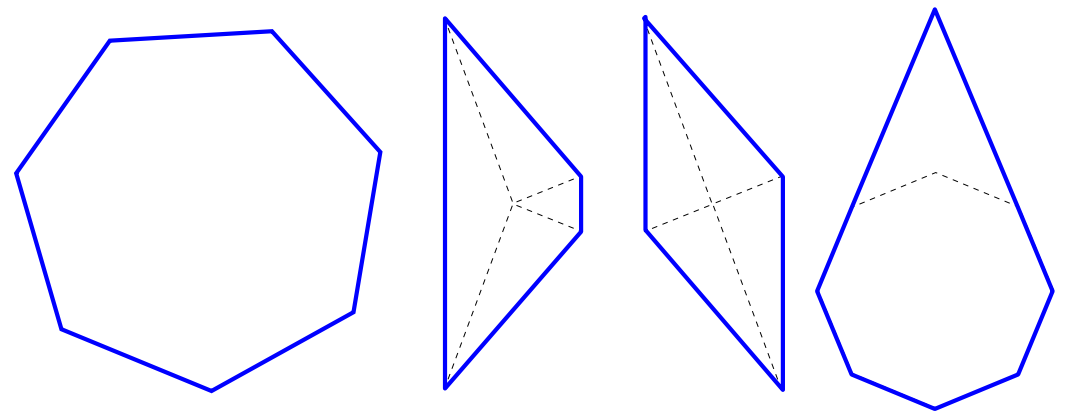

Fig. 5. Left to right, shapes preserved by the CHASE dynamics: regular polygons, trapezoids, rhombi, and drops. In pictures of rhombus and trapezoid, the dashed lines are the bisectors.

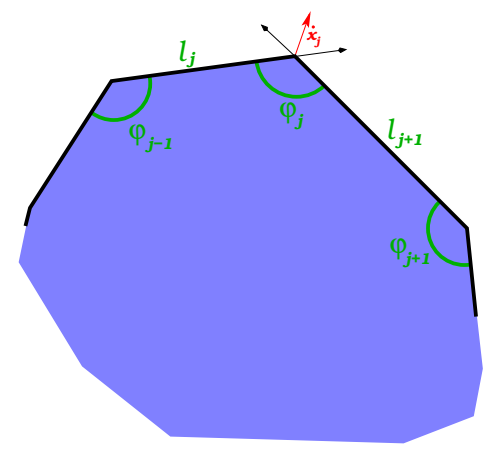

Fig. 6. Notations for Theorem 2

and, similarly, the rates for the lengths $\ell_{j}$ :

$$
\dot{\ell}_{j}=2+\cos \varphi_{j}+\cos \varphi_{j-1} \text {. }
$$

If the shape of the polygon remains invariant, the angles are constant. This implies a system of linear equations on $s_{j}:=\sin \varphi_{j}$ 's. It is immediate that this system is identical to the conditions on stationary probabilities on a continuous time Markov chain on the circular graph with transition rates $1 / \ell_{j}$. As this auxiliary Markov chain is manifestly ergodic, such stationary probabilities are unique and therefore the solutions are spanned by the vectors of $\left(s_{j}\right)_{j}=(1,1, \ldots, 1)$. Hence, all $\sin \varphi_{j}$ are equal, and that therefore for some $\varphi$, all of $\varphi_{j}$ are equal to either $\varphi$ or $\pi-\varphi$.

We note that this condition implies that one of the following holds:

- There are 3 acute angles, forcing the polygon to be a regular triangle;

- or there are 2 acute angles equal to $\varphi<\pi / 2$, and two complementary angles $\pi-\varphi$, forcing the polygon to be either a trapezoid or a rhombus;

- or there is one acute angle $\varphi$, and all the remaining angles are equal to $\pi-\varphi$, or

- all angles are obtuse. 
The 4-sided polygons are easily seen to be realizable with the angle $\varphi$ being a continuous shape parameter.

As for the last two options, the preservation of the shape implies that the ratios of the lengths $\ell_{j} / \ell_{j+1}$ remains constant, and therefore equal to the ratios of the $\dot{\ell}_{j} / \ell_{j+1}$. Combining this with the equation (4) implies that the ratios of the lengths $\ell_{i} / \ell_{j}$ can take only following values (in case of one acute angle)

$$
1, \frac{1 \mp \cos \varphi}{1 \pm \cos \varphi},(1 \pm \cos \varphi)^{ \pm 1} .
$$

This immediately lead to the conclusion that only drop shape can satisfy this condition.

As for the case where all the angles are equal, the equality of all sidelengths is obvious.

\subsection{Regular polygons are unstable}

As we discussed in section 2.5, the space of shapes of $k$-sided polygonal chains is naturally isomorphic to the $k$-2-dimensional complex projective space and has therefore (real) dimension $2 k-4$.

A natural coordinate frame on a (everywhere dense) chart in this space is given by the lengths of the sides of the $k$-gon, normalized to $\sum \ell_{j}=1$ (this gives $k-1$ coordinates) and collection of angles $\varphi_{j}$ satisfying the condition $\sum \varphi_{j}=(k-2) \pi$. The condition of closing the polygon implies that only $(k-3)$ of these angles are independent.

Under the CHASE dynamics the vertices of the $k$-sided polygon move according to the equation (2). The next proposition shows that the regular $k$-sided polygon, while a fixed point of CHASE dynamics, is unstable in linear approximation.

In the next proposition we talk about the linearization of the CHASE dynamics on the shape space.

Remark that the vector field CHASE there is defined only up to a multiplication by a positive smooth function. However, one can readily verify that the linearization of the vector field at an equilibrium point is unaffected by this ambiguity.

Theorem 3 The linearization of the CHASE dynamics near the k-regular polygonal chain the $2 k-4$-dimensional space of $k$-sided shape has exactly $(k-3)$-dimensional stable subspace (tangent to the manifold $\{\varphi=$ const $\}$ and $(k-1)$-dimensional invariant subspace, unstable for $k \geq 5$.

Proof. One can check immediately that under the CHASE evolution, the sides of the $k$-gons with all angles equal (to $\pi(1-2 / k)$ ) move parallel to themselves. This proves the invariance of $\{\varphi=$ const $\}$, and also the fact that (when considered in the space of $k$-sided polygons), the lengths are all growing linearly, with the same speed. Therefore, their ratios asymptotically tend to 1 , proving the first claim.

It follows that in the $(\ell, \varphi)$ frame on conf, the linearization of the CHASE vector field is block-upper-triangular: 


$$
J=\left(\begin{array}{cc}
J_{\ell} & \ddots \\
0 & J_{\varphi}
\end{array}\right)
$$

Using (3) we compute $J_{\varphi}$, to obtain a cyclic tri-diagonal matrix:

$$
J_{\varphi}=-\frac{2 \cos \left(\frac{(k-2) \pi}{k}\right)}{\ell}\left(\begin{array}{rrrrrr}
2 & -1 & 0 & \cdots & 0 & -1 \\
-1 & 2 & -1 & 0 & \cdots & 0 \\
0 & -1 & 2 & -1 & \cdots & 0 \\
\vdots & & \ddots & \ddots & \ddots & \vdots \\
0 & \cdots & 0 & -1 & 2 & -1 \\
-1 & 0 & \cdots & 0 & -1 & 2
\end{array}\right)
$$

whence

$$
\operatorname{spec}\left(J_{\varphi}\right)_{j}=-\frac{4 \cos \left(\frac{(k-2) \pi}{k}\right)}{\ell}\left(1-\cos \left(\frac{2 \pi j}{k}\right)\right)
$$

which belongs to $(0, \infty)$ if $k \geq 5$. This, clearly, proves the claim.

\section{Stability of triangles}

A corollary of the proof of Theorem 3 implies that the regular triangles, under the CHASE dynamics are stable. This result is, however, relatively useless, as the Markov chain $\mathbf{P}_{t}$ is rather different from the tamed dynamics (which is approximated by CHASE ): the triangles acquire extra vertices, and, while the growth of the resulting polygons might or might not be slowed down compared to the tamed process, we are missing currently tools to analyze it.

For this reason we present a simple analysis of somewhat more general than CHASE dynamics, where the averaged speed of an apex at $j$-th point $(j=1,2,3)$ has a outward velocity $v=v\left(\varphi_{i} / 2\right)$ directed along the bisector, but having a general form (not necessarily $2 \cos \varphi / 2$, as in the case of CHASE).

We will write

$$
\alpha=\varphi_{1} / 2, \beta=\varphi_{2} / 2, \gamma=\varphi_{3} / 2
$$

As above, we find

$$
\dot{\alpha}=\frac{1}{\sin \beta}(v(\gamma) \sin \gamma-v(\alpha) \sin \alpha)+\frac{1}{\sin \gamma}(v(\beta) \sin \beta-v(\alpha) \sin \alpha)
$$

Denote $g(\alpha)=v(\alpha) \sin \alpha$. Then, condition on stationary point gets the form

$$
g(\alpha)=g(\beta)=g(\gamma) .
$$

Differentiating (5) with respect to $\alpha$ and $\beta$ we obtain linear stability condition on stationary point. Let $J=\left(J_{i, j}\right)$ be the linear part of $[5$.

Then the linear stability conditions have the form

$$
0 \geqslant \operatorname{tr}(J)=-\left(g^{\prime}(\alpha)\left(\frac{1}{\sin \beta}+\frac{1}{\sin \gamma}\right)+g^{\prime}(\beta)\left(\frac{1}{\sin \gamma}+\frac{1}{\sin \alpha}\right)+g^{\prime}(\gamma)\left(\frac{1}{\sin \alpha}+\frac{1}{\sin \beta}\right)\right)
$$


and

$0 \leqslant \operatorname{det}(J)=\left(g^{\prime}(\alpha) g^{\prime}(\beta)+g^{\prime}(\beta) g^{\prime}(\gamma)+g^{\prime}(\gamma) g^{\prime}(\alpha)\right)\left(\frac{1}{\sin \alpha \sin \beta}+\frac{1}{\sin \beta \sin \gamma}+\frac{1}{\sin \gamma \sin \alpha}\right)$.

In particular, if $g^{\prime}(\pi / 3)>0$, the regular triangles are stable.

\section{Conclusions}

\subsection{Summary}

Summarizing, the results we presented lend theoretical support to the observed experimentally phenomena: the CHASE dynamics which is approximating the original Markov chain well for large polygons near $k$-sided regular ones for $k \geq 5$ is unstable. While we do not know what is the correct approximation for the polygons close (in some sense) to large triangles, a reasonable CHASE -like approximation is stable near regular triangles.

Clearly, the problem of proving the asymptotic symmetry breaking is still open.

\subsection{Higher dimensions}

One can look at the analogous problem in higher-dimensional setting. Experiments show that the convex hulls of RRTs in $\mathbb{R}^{d}$ form approximately a regular $d$-simplex. We do not have, yet, any results similar to our planar case.

\subsection{Case $k \rightarrow \infty$}

Let us now consider limiting case for the infinitely many vertices of initial shape.

Without going into details, we just remark here, that in a natural parametrization, the CHASE evolution is described in this limit by the one-dimensional Boussinesq equation

$$
\dot{\varphi}=\left(\varphi^{2}\right)^{\prime \prime} .
$$

\section{References}

1. M. Ballerini, N. Cabibbo, R. Candelier, A. Cavagna, E. Cisbani, I. Giardina, V. Lecomte, A. Orlandi, G. Parisi, A. Procaccini, M. Viale, and V. Zdravkovic. Interaction ruling animal collective behavior depends on topological rather than metric distance: Evidence from a field study. Proc. National Academy of Sciences, 105:1232-1237, 2008.

2. Yuliy Baryshnikov and Vadim Zharnitsky. Sub-Riemannian geometry and periodic orbits in classical billiards. Math. Res. Lett., 13(4):587-598, 2006. 
3. D. Hsu, L. E. Kavraki, J.-C. Latombe, R. Motwani, and S. Sorkin. On finding narrow passages with probabilistic roadmap planners. In et al. P. Agarwal, editor, Robotics: The Algorithmic Perspective, pages 141-154. A.K. Peters, Wellesley, MA, 1998.

4. D. Hsu, J.-C. Latombe, and R. Motwani. Path planning in expansive configuration spaces. International Journal Computational Geometry \& Applications, 4:495-512, 1999.

5. S. Karaman and E. Frazzoli. Sampling-based algorithms for optimal motion planning. International Journal of Robotics Research, 30(7):846-894, 2011.

6. Allen Knutson. The symplectic and algebraic geometry of Horn's problem. Linear Algebra Appl., 319(1-3):61-81, 2000. Special Issue: Workshop on Geometric and Combinatorial Methods in the Hermitian Sum Spectral Problem (Coimbra, 1999).

7. H. J. Kushner and G. G. Yin. Stochastic Approximation and Recursive Algorithms and Applications. Springer Verlag, 2003.

8. F. Lamiraux and J.-P. Laumond. On the expected complexity of random path planning. In Proceedings IEEE International Conference on Robotics \& Automation, pages 33063311, 1996.

9. S. M. LaValle. Rapidly-exploring random trees: A new tool for path planning. TR 98-11, Computer Science Dept., Iowa State University, October 1998.

10. S. M. LaValle. Robot motion planning: A game-theoretic foundation. Algorithmica, 26(3):430-465, 2000.

11. S. M. LaValle. Planning Algorithms. Cambridge University Press, Cambridge, U.K., 2006. Also available at http://planning.cs.uiuc.edu/.

12. O. Nechushtan, B. Raveh, and D. Halperin. Sampling-diagram automata: A tool for analyzing path quality in tree planners. In Proceedings Workshop on Algorithmic Foundations of Robotics, Singapore, December 2010.

13. Herbert Robbins and Sutton Monro. A stochastic approximation method. Ann. Math. Statistics, 22:400-407, 1951.

14. J. Yu, D. Liberzon, and S. M. LaValle. Rendezvous wihtout coordinates. IEEE Transactions on Automatic Control, 57(2):421-434, 2012. 